\title{
New challenges with the Journal of Plant Research
}

\author{
Ikuo Nishida
}

Published online: 23 December 2014

(C) The Botanical Society of Japan and Springer Japan 2014

This is the second new year for me as the Editor-in-Chief of the Journal of Plant Research (JPR). In the last year, the Botanical Society of Japan has decided to increase the global credibility of JPR under the support of the Japan Society for the Promotion of Science (Nishida 2014). Our missions are condensed in the following four key phrases: (1) rapidness of publication, (2) reader-friendly presentation, (3) dissemination of highly qualified scientific findings, and (4) archiving of interdisciplinary knowledge in plant science. The first three missions have been successfully approaching their goals under the strong support of the Editors, Editorial Board members, anonymous reviewers, Editorial Office staff, and the Springer team members. Compared with these missions, the fourth one is a new challenge for us: I would like to inform you that JPR and Ecological Research (ER; Springer) have agreed to issue the special virtual issues "Joint contents from the Journal of Plant Research and Ecological Research" (http://springer.com/10265). The first issue, entitled "Interdisciplinary approach for spatial and temporal dynamics of carbon cycle processes in terrestrial ecosystems: Challenges and networking at the Takayama site on a mountainous landscape of Japan", has been launched by Dr. Hiroyuki Muraoka as an Editor.

At the start of the new year, I am very pleased to announce that this issue begins with a JPR symposium entitled "Plasmodesmata: Function and Diversity in Plant Intercellular Communication", organized by Prof. Tomonichi Fujita. Plasmodesmata are unique organelles in plants and algae and function as conduits for nutrients, signaling

I. Nishida $(\bowtie)$

Division of Life Science, Graduate School of Science and Engineering, Saitama University, 255 Shimo-Okubo, Sakura-Ku, Saitama 338-8570, Japan

e-mail: jpreic@gr.saitama-u.ac.jp molecules, some macromolecules, and even viruses. One of the unique features of this symposium is that it focuses on not only the function but also the diversity of plasmodesmata, which is sure to attract the interest of researchers across the breadth of plant sciences. Contributors to this symposium include four young scientists, from whom I anticipate future submissions to JPR of their excellent studies. I would like to acknowledge Prof. Masayoshi Kawaguchi, who took the role of Editor in this symposium.

Another JPR symposium, entitled "Reprogramming of Plant Cells as Biological Strategies", is scheduled for a coming issue this year under the supervision of Drs. Keiko Sugimoto and Masaki Ito. This symposium is based on original presentations in the symposium organized by the young talented scientists Dr. Satoru Iwase and Momoko Ikeuchi at the annual meeting of the Botanical Society of Japan (13-15 September 2013 at Hokkaido University, Sapporo). Cell reprogramming is regarded as a survival strategy of plants, the sessile organism. To cope with changing environmental conditions, plants are able to change the fates of developing and dividing cells or even determined cells by a process called reprogramming and dedifferentiation. I hope you will enjoy being at the frontier of this new field.

Our editorial policy states that JPR provides opportunities for publishing across the breadth of plant sciences (Nishida 2013). The research areas range widely, i.e., from taxonomy, phylogenetics, and evolutionary biology to ecology and ecophysiological and environmental biology; morphology, anatomy, and structural biology; genetics and developmental biology; physiology, biochemistry, and molecular and cell biology; and other, related fields. Thus, all authors are encouraged to prepare their manuscripts with consideration for understanding by a readership outside their own research fields. To guarantee the originality 
and quality of accepted articles, we would like to welcome three new Editors for a 2015-2018 term, Drs. Atushi Ebihara, Yoko Kakugawa-Yatabe, and Rhett D. Harrison. I would like to acknowledge continued support from the current Editors, Drs. Kouki Hikosaka, Masayoshi Kawaguchi, Masaki Ito, Shuichi Sakaguchi, Maki Katsuhara, Shinobu Satoh, and Hideo Yamasaki. Decisions by the Editors are supported by the critical but constructive comments of diverse Editorial Board members and a large number of reviewers. Graph presentation quality is checked by Junko Kadkhodaei, who also dedicates herself to the efficient running of the Journal of Plant Research Editorial office. Our Editorial coordinator, Miles Peterson, whose work has already been appreciated by many authors, does a final brush-up of the articles. The highly professional support by the Springer team, especially Francina Sebastian, Yuko Matsumoto, and Aiko Hiraguchi, is also to be acknowledged for improving the status of JPR in plant sciences. During the last 2 years, the journal's impact factor has been rising to record its best score, 2.507 (Nishida 2014), and this encourages and convinces us to continue our editorial policy in 2015.

We welcome new Editorial Board members who begin their 4-year terms in January 2015: Drs. Masahide Yamato, Ze-Long Nie, Wenheng Zhang, Tod Stuessy, Kentaro Shimiyu, Fei-Hai Yu, Shuang-Quan Huang, Koichi Takahashi, Toshihiko Kinugasa, Shinichi Miyazawa, Mitsuru Hirota, Richard Whitkus, Goro Horiguchi, Kimitsune Ishizaki, Chandrashekhar Joshi, Laigaeng LI, Edouard Pesquet,
Byung-Ho Kang, Nina Kamennaya, Alex Costa, Kensaku Suzuki, and Charls P. Chen.

Special thanks are to be given to Profs. Akiko Soejima and Toshihiro Yamada, who have done excellent jobs as Editors in the areas of taxonomy, phylogenetics, and evolutionary biology for the last 4 years. We also thank those who rotate off the Board including Drs. Atsushi Ebihara, Yoko Kakugawa-Yatabe, Seung-Chul Kim, Yuko T. Hanba, Jin-Sheng He, Nobukazu Kakagoshi, Yanhong Tang, Atushi Ushikaru, Shiro Tsuyuzaki, Taku Demura, Herman Höfte, Toshinori Kinoshita, Jun'ichi Mano, and Chikahiro Miyake as well as a Springer team member, Momoko Asawa.

Nominations for the JPR 2015 Awards will be welcome in early January 2015, and all JPR authors are eligible. We wish you a Very Happy New Year and we welcome submissions of excellent articles in 2015.

Ikuo Nishida

Editor-in-Chief, Journal of Plant Research

\section{References}

Nishida I (2013) From the new Editor-in-Chief: a sense of personal identity with the Journal of Plant Research. J Plant Res 126:321-322

Nishida I (2014) As we begin a new year with the Journal of Plant Research. J Plant Res 127:1-2 